\title{
Exploration and Analysis of the Integrated Enterprise Marketing in the Context of Microfilms and New Media
}

\author{
Shikun Liu \\ College of Media and Communication, Zaozhuang University
}

\begin{abstract}
This paper is aimed at allowing enterprises to take full advantage of microfilms for the integrated marketing in the fierce market competition in the context of new media, so that product information can be kept in the heart of customers, and further customers can give priority to their products for satisfying purchase demand. This paper analyzed the enterprises' mode of integrated marketing in the context of new media and emphasized on studying how to combine microfilms with the integrated enterprise marketing to achieve the optimal promotion effect through the cases, presented the combination mode of microfilms and integrated enterprise marketing, and forecasted the future development trend.
\end{abstract}

Keywords-Newmedia; Microfilms; Enterprise; Integrated marketing

\section{INTRODUCTION}

In the wake of the popularization of the mobile intelligent terminal and small high-definition equipment, and the development of online network videos, the hot wave of microfilms has been spread all over the world, a plenty of excellent works emerged, and various video websites also cooperated with various enterprises successively to perform all kinds of microfilm production plans within the second ten years of the 21 st century. Especially with the fast rising of Kuaishou, Tik Tok, Huoshan Video and other short video platforms, the micro-video received the affection from more and more audiences on the new media platform with its new audio and visual pattern, rich subject selection, flexible video production methods and diversified viewing channels. As for one enterprise, the marketing is the priority among priorities. One enterprise shall constantly integrate its marketing strategies with various tools and means to satisfy the needs of the current market and the new age under the new media context, and therefore it is one of the important topics for the micro-video development and the enterprise marketing that how to better blend the microfilms with the enterprise's integrated marketing under the new age to achieve the optimal promotion effect.

\section{INTEGRATED ENTERPRISE MARKETING MODE IN THE CONTEXT OF NEW MEDIA}

In recent 10 years, the handheld intelligent terminal devices represented by tablet computer and mobile phone have caused the mobile internet revolution, especially in the wake of the popularization of domestic mobile $4 \mathrm{G}$ internet and the public spread of WIFI network since 2012, it is reported from the 42th China Internet Network Development State Statistics Report issued by China Internet Network Information Center (CNNIC) that up to June 30, 2018, the number of cyber citizens in China has reached 802 million, including 788 million mobile cyber citizens. While the user cohesion has also become increasingly higher with the popularization of mobile payment and other new payment means. There are numerous users attracted into the army of new media with the rapid development of "Otaku" culture, the MicroBlog, WeChat and other SNS social media platforms, and the online video platforms represented by Youku and IQiyi.

Currently, an increasing number of enterprises have realized the important promotion role of new media, started to greatly reduce the traditional offline public relations and marketing activities, and transferred more effort to the new media platform. The image promotion, product release and even the guidance of public opinion conducted by the enterprises as the business entity of market economy based on its development strategy and profit objective have become the new promotion direction for the enterprise strategies, especially for some products targeted to the young user group, such as electronic products, clothes, household passenger vehicles and sports equipment. The pattern of enterprises' marketing position becomes more complex with the continuous improvement of some professional forums, the popularization of WeChat official account number, MicroBlog and other new social media platforms, and the emerging of new de-massified gathering communities represented by Zhihu. It has become the optimal choice of marketing and development for the enterprises to follow the new tendency of intreated marketing communication and take full advantage of diversified new media as the most important communication tools. The enterprises' marketing promotion also showed the differentiated features by virtue of different media patterns. 
The official website of one enterprise as the most important position for building enterprise image is vital, but its official MicroBlog, WeChat official account and other new social media platforms can display the dynamic news, product update and other contents more quickly and in real time, and enterprise can utilize the new media to interactively collect the user feedback and public opinion, publish the video advertisement with the focus on the product experience at the online video websites with the greatest concentration of users such as the carpet bombing at Youku, IQiyi and other websites. While enterprises conduct the relative professional and flexible enterprise promotion for its product introductions at the relatively professional community forums such as Douban, Zhihu and Tianya, what's more, they can conduct the guidance of public opinion via these relative professional communities, thus resulting in the second information transfer. Such cases are too numerous to mention one by one, for example, it was exposed frequently that the newly released film is refreshed for higher or lower score maliciously at some famous website, another example is that domestic mobile phone manufacturers hire the online water army for the ferocious battle in the public opinion such that many enterprises straightforwardly launched the sub-brands mainly aimed at the online marketing, such as Leader series household electrical appliances of Haier. The enterprises achieved the combined communication by the aforementioned means, and it is the most important pattern of integrated enterprise marketing under the new media context at present.

\section{ADVANTAGES OF MICROFILMS IN THE INTEGRATED ENTERPRISE MARKETING}

In the field of films and television, the microfilm marketing customized for one enterprise or its products when compared with the relatively rigid and traditional product placement advertisements is the brand-new means for the integrated enterprise marketing. Enterprises will integrate its products into stories and enable audiences to immerse in the wonderfully dramatic or deeply moving scenario to arouse the emotional resonance and the imperceptible identity with the story line, the identity with the emotion and life style of features in the stories, and final identity with the products in a more gentle manner, rather than directly push products to audiences by the rude approach.

As one enterprise marketing means, the microfilm has its unique advantages. First, compared with the huge size of traditional films, the microfilm is relatively small in the aspects of capital input, staff composition, shooting equipment and production process. The technical threshold for the microfilm production becomes increasingly low with the price reduction of small-size high-definition shooting equipment and the popularization of digital single-lens reflex equipment. Even some enterprises shot microfilms with their own products, for example, the microfilms released by the manufacturers of Canon camera, Sony mirrorless camera and other digital equipment in recent several years are mostly shot by these products promoted in the microfilm, it is not only the selfconfidence for their products, but also a new marketing method. Second, Microfilm is more attractive and ornamental. It has been very difficult to attract the attention from audiences for the traditional enterprise promotion film or product advertisement due to its extremely obvious purpose of persuasion and indoctrination, simple or even rare story line. While there are wide subject matters, many relatively large production teams and even popular celebrities involved in the microfilms, it is undoubted to attract more attention from audiences. For example, the thriller and suspense microfilm Touch and Go jointly produced by Cadillac and Yanzu WU caused the great influence at that time. Another example is that " Four Nights Odd Tan" series jointly produced by Sina and Samsung Electronics, "7 Movies - Actors into Directors" jointly produced by Sohu Video and Nokia, which presented a series of great films such as Before the Ocean Current, Blue Tears and A Nail Clipper Romance. Third, the microfilm is the same as MicroBlog and other new media, namely that it is easier for a small enterprise to change its line of products, and has the features of timely receiving the information feedback and updating. The hot news events would come in the rush and attract the attention from whole network, but then slip away just like tidewater under the current network onlookers and the orgiastic age, and the cyber citizens are longing for more new news events. How to make the hot events together with the enterprise or product raise the public concern became one of the most important steps in the enterprise marketing, hence "Event Marketing" became a kind of time-tested magic weapon. As for these real hot events with the negative social influence, enterprises can express its opinion and undertake the required social liabilities via microfilms or by other methods. For example, the microfilm Unclassified Death directed by Mo ZHANG in the "7 Movies - Actors into Directors" series jointly produced by Sohu Video and Nokia, the film is featured by highly dramatic role design, exaggerated performance and symbolization, and describes the store with high dramatic tension through the event that one old man falls down to the ground inside one fast food restaurant, but no one dares give his helping hand. The film is just the powerful response to the most controversial event at that time that the elder fallen down to the ground when crossing the road, but not one dared give his helping hand. Fourth, the audience group of the microfilms is basically in coincidence with the middle force of social consumption, the audiences mostly concentrate in the young generations after $70 \mathrm{~s}, 80 \mathrm{~s}$ and $90 \mathrm{~s}$, and they are also a force with the highest consumption potential. Such group shows their apparent preference to microfilms in the film form of fragmented reading, and the group has a relatively high tolerance level for these flexible advertisements, and even can consciously seek for the identity at the same time of accepting the brand marketing. 


\section{COMBINATION BETWEEN MiCROFILM AND INTEGRATED ENTERPRISE MARKETING}

The microfilms have appeared the flourishing situation since its initial development of "Spoof" mode, all kinds of microfilms with excellent creativity and strict story line have become available in succession such that it not only becomes the cultural tool for public amusement, but also the important carrier of enterprises for the product promotion. Presently, the microfilm production agencies get the profit sharing of advertisements through the cooperation with online video websites or get the profits directly through the cooperation with enterprises, online video websites and film distribution corporation. In this background, the microfilm production agencies of all sizes were established all over the country, and the microfilm industry has begun to take shape. As for enterprises, the microfilm marketing is not only one means for the product promotion, but also even one magic weapon for building the enterprise image. Some world-famous enterprises would usually present the relevant microfilms to further enhance the orientation, novel properties, etc. of product except the overwhelming hard advertisements at the time of releasing new product, especially the enterprises or brands targeted to the young people, for example, the FAW-Volkswagen presented the Babylon Misty at the time of listing new CC, the microfilm shaped the elegance and refinement different from that of previously released cars, which was widely broadcasted at Youku, Tencent Video, Sohu Video, IQiyi and other mainstream video platforms and quickly attracted the considerable attention and sharing on the Internet by virtue of its science fiction, suspense, action, fashion and other many elements. Microfilm is not only required for marketing new product released, even though the enterprise's other activities but also can be expressed by the microfilm to attract the attention from relevant audiences. For example, the inspirational microfilm Stubbornness produced by Huawei for employing the current year's college graduates, which not only attracts the attention of relevant professional talents on Huawei and its recruitment, but also can reveal the youthful \& positive and courageous \& fighting enterprise image within the larger scope, thus further leading the sales volume of relevant products made by Huawei. Moreover, even though there is no new product released, these enterprises adept in the integrated marketing can also present the relevant microfilms to reinforce their sense of presence and attract the attention of audiences at some important time nodes. The New Year microfilm series, including the Transfer of Love, Bring Happiness Home, Come Together, Monkey King Family, etc. with the focus on the reunion and tender feeling presented by Pepsi since 2012 are relatively influential that the most popular movie and television stars in the current year are often invited for cheering. Larger sports brand companies can produce some microfilms endorsed by their own sports stars during the period of important sports events, for example the Football Legend jointly presented by Nike and dozens of sports stars prior to the World Cup and Don't Stop Me Now presented by Adidas during the Olympic Games in London obtained the continuous attention in the large-scale events and shaped the strong sports brand image, and their attraction given from young people is self-evident.

\section{CONCLUSIONS}

The microfilm is the newly sprouted thing in the new era, its production, propagation and receiving are based on the network platforms. Compared with the traditional film works, publications, etc., there is no very strict shooting license, examination and other mechanisms related to such cultural products currently at the state level such that the microfilms and other network-based cultural products obtain the very loose creation environment, but the barbaric growth of microfilm industry is caused just due to low threshold for its investment, shooting and propagation, and there are numerous poor works emerged due to the absence of industry self-regulation. While the continuous improvement of human aesthetic taste, various online video websites competitively seek for the traditional high-quality movie resources, in such case, the viewing rate competition of online videos becomes white-hot, therefore the microfilm must strive for improving its self-quality in order to share this cake. With the diversified development of integrated enterprise marketing, it is apparent that the microfilm isn't the only choice for the integrated enterprise marketing, hence the further development tendency of microfilms in the integrated enterprise marketing is to better improve the self-quality of microfilms, and build the brand advantages, thus multidimensionally and multifariously combining with the integrated enterprise marketing under the grand tide of new media and reaching the cooperation mode of mutual benefit and win-win.

\section{REFERENCES}

[1] Discussion on the Status and Development Trend of Chinese Microfilms Yiying ZHOU Modern Business 2014.

[2] Research on the Communication of Real Estate Integrated Marketing in the New Media Era - Take an Example of Longfor Properties Modern Enterprise Culture Chenziyu GUO 2011.

[3] Brief Analysis on the Microfilm-based Marketing Strategies for Brand Communication in the New Media Era Yu SONG Today's Mass Media 2014. 